\title{
A Full-Vectorial Pseudospectral Modal Analysis of Dielectric Optical Waveguides With Stepped Refractive Index Profiles
}

\author{
Chia-Chien Huang, Chia-Chih Huang, and Jaw-Yen Yang
}

\begin{abstract}
A new full-vectorial mode solver based on the pseudospectral method for computing dielectric optical waveguides is proposed. The subdomains with homogeneous refractive indexes are connected with imposing the continuities of $E_{z}$ and $H_{z}$ at the dielectric interfaces, and thus the coupled equations in terms of the transverse magnetic field components $H_{x}$ and $H_{y}$ are formulated. The optical fields are expanded by a suitable set of orthogonal basis functions, such as Laguerre-Gauss (LG) functions or Chebyshev polynomials. Furthermore, the a priori determination of the scaling factor in LG functions is introduced by means of the effective index method in order to considerably reduce the extra computational time by lengthy trial and errors used by others. Results calculated by the proposed full-vectorial scheme for dispersion characteristics of rectangular channel and rib waveguides having piecewise uniform refractive indexes, even though a coarse mesh used, show good agreement with previously published data based on other rigorous numerical methods.
\end{abstract}

Index Terms-Dielectric optical waveguide, full-vectorial mode solver, multidomain, orthogonal basis function, pseudospectral method, rectangular channel waveguide, rib waveguide.

\section{INTRODUCTION}

$\mathbf{M}$ ODAL analysis for optical waveguides is an essential issue for simulating the performances of diverse photonic integrated circuits through the information of propagation constants and field distributions of guided modes. A number of numerical and approximate methods have been deeply reviewed by Chiang [1]. Among them, the rigorous numerical methods in general cover three groups: the finite difference (FD) [2], [3], finite element (FE) [4], [5], and series expansion methods [6]-[9]. Recently, the series expansion methods have emerged as a powerful mode solver due to their fewer requirements of memory storage for a smaller matrix and better efficiency for obtaining highly accurate results compared with the FD and FE methods. In [6] and [7], a novel Hermite-Gauss (HG) orthogonal collocation method (also termed the pseudospectral method [10], [11]) with the usual single domain based on scalar wave equation was proposed to solve propagation characteristics of modes

Manuscript received July 13, 2004; revised February 5, 2005. This work was supported by the National Science Council, Taiwan, R.O.C. under Contract NSC 93-2215-E-275-001.

C.-C. Huang is with the Department of Information Management, Ling Tung College, Taichung 408, Taiwan, R.O.C. (e-mail: cgs1120@ms34.hinet.net).

C.-C. Huang is with the Department of Electronic Engineering, Tung Nan Institute of Technology, Taipei 222, Taiwan, R.O.C.

J.-Y. Yang is with the Computational Electromagnetics and Plasma Lab., Institute of Applied Mechanics, National Taiwan University, Taipei 10617, Taiwan, R.O.C.

Digital Object Identifier 10.1109/JSTQE.2005.846540 for planar waveguides and to research propagating waves for tapered waveguide structures. The orthogonal collocation method achieves the equal convergent rate comparing with Galerkin's method [8], [9] that usually demands considerable evaluation of laborious integral elements, but it needs much less computational effort, as proved by experimental data in [7]. However, while encountering discontinuous refractive index profile (RIP) structures, the values usually oscillate about the exact solutions and show relatively poor convergence [7]. As a result, the great advantages of rapid convergence and high accuracy by the pseudospectral method are therefore lost.

Moreover, the scaling factor in HG used by [7] was determined by extra trial and error to experiment with it. In order to deal with these issues, our previous work [12] using multidomain pseudospectral method (MPM) formulated by scalar wave equation has been presented and the results tested by a number of slab and three-dimensional (3-D) diffused waveguide structures show excellent agreement with exact analytical solutions or the published values by other numerical schemes. On account of the assurance, the purpose of this paper is to extend the work in [12] considering the full-vectorial formulations based on $H_{x}$ and $H_{y}$. The full-vectorial wave equations for the MPM are initially described in Section II. In Section III, the main solution techniques are stated in detail including patching conditions used, the choice of basis functions, and the determination of the scaling factor. Section IV presents numerical examples and discusses the results compared with others. Finally, some conclusions are made in Section V.

\section{MATHEMATICAL FORMULATION}

The modal characteristics of 3-D dielectric waveguide structures are, in general, hybrid. For the accurate representation of the features of electromagnetic waves, the full-vectorial formulations are required. Assuming a monochromatic electromagnetic field propagating along the direction in an inhomogeneous medium with refractive index $n(x, y, z)$, the vector wave equation derived from Maxwell's equations for a source free region in the frequency domain based on the magnetic field vector $\mathbf{H}$ is given by

$$
\nabla^{2} \mathbf{H}+k_{0}^{2} n^{2} \mathbf{H}+\frac{\nabla n^{2}}{n^{2}} \times(\nabla \times \mathbf{H})=0
$$

where $k_{0}=2 \pi / \lambda_{0}$ and $\lambda_{0}$ is the wavelength in vacuum. In regard to the guided mode problems, the longitudinally invariant structure is usually considered. Consequently, the variation of 
the refractive index depends only on the transverse directions, and we have $n=n(x, y)$. The desired coupled wave equations in terms of $H_{x}$ and $H_{y}$ for solving guided mode problems are thus obtained as shown in (2) and (3) at the bottom of the page, where $n_{\text {eff }}=\beta / k_{0}$ denotes the mode effective index and $\beta$ is the propagation constant along the $z$ direction. The pseudospectral method with the single domain obtains highly accurate and efficient solutions while the RIP is continuous in the whole computational domain, but it is not the case for discontinuous RIP [7]. However, optical waveguide structures generally involve a number of discontinuous RIP, for instance, channel and semiconductor rib waveguides, etc. [13]. To alleviate the severe problem, we use the domain decomposition approach to partition the interest domain into a number of subdomains with uniform refractive index. Under this consideration, the polarization dependence and the coupling terms in (2) and (3) for each subdomain are hence gone. The wave equations are reduced as the following:

$$
\frac{\partial^{2} H_{x}}{\partial x^{2}}+\frac{\partial^{2} H_{x}}{\partial y^{2}}+k_{0}^{2}\left(n^{2}-n_{\mathrm{eff}}^{2}\right) H_{x}=0
$$

and

$$
\frac{\partial^{2} H_{y}}{\partial x^{2}}+\frac{\partial^{2} H_{y}}{\partial y^{2}}+k_{0}^{2}\left(n^{2}-n_{\text {eff }}^{2}\right) H_{y}=0 .
$$

The full-vectorial formulations (4) and (5) based on MPM seem to be decoupled. However, by incorporating interfacial conditions between subdomains, they can be recovered to a set of coupled equations as shown below.

In (4) and (5), $H_{x}$ and $H_{y}$ at each intraelement boundary are continuous for both horizontal and vertical interfaces of different materials. In addition, the continuities of the longitudinal components $H_{z}$ and $E_{z}$ across dielectric interfaces can incorporate the coupled relations of $H_{x}$ and $H_{y}$ through both $\nabla \times \mathbf{H}=$ $j \omega \varepsilon_{0} n^{2}(x, y) \mathbf{E}$ and $\nabla \cdot \mathbf{H}=0$. First, we consider a horizontal interface as depicted in Fig. 1(a). According to the continuities of both $H_{z}$ and $E_{z}$ at interfaces and the derivatives of $H_{x}$ and $H_{y}$ with respect to $x$ at both sides of the interface are equal, the continuity of $E_{z}$ yields

$$
\left.n_{y+}^{2} \frac{\partial H_{x}}{\partial y}\right|_{y-}-\left.n_{y-}^{2} \frac{\partial H_{x}}{\partial y}\right|_{y+}=\left(n_{y+}^{2}-n_{y-}^{2}\right) \frac{\partial H_{y}}{\partial x}
$$

and the continuity of $H_{z}$ gives

$$
\left.\frac{\partial H_{y}}{\partial y}\right|_{y+}=\left.\frac{\partial H_{y}}{\partial y}\right|_{y-}
$$

where $y+$ and $y-$ are referred to the locations at the infinitesimally upper and lower sides of the horizontal interface, respec-

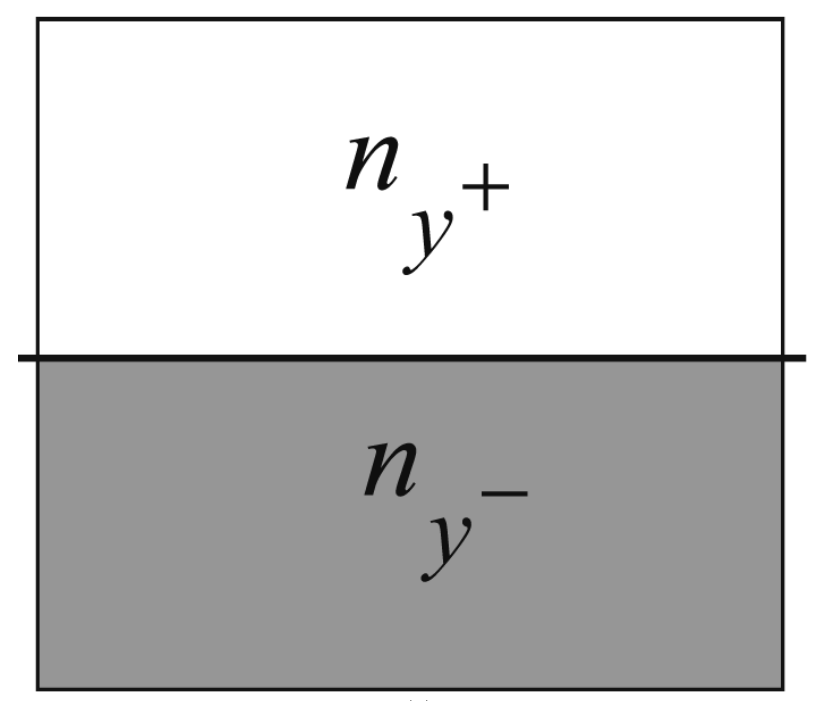

(a)

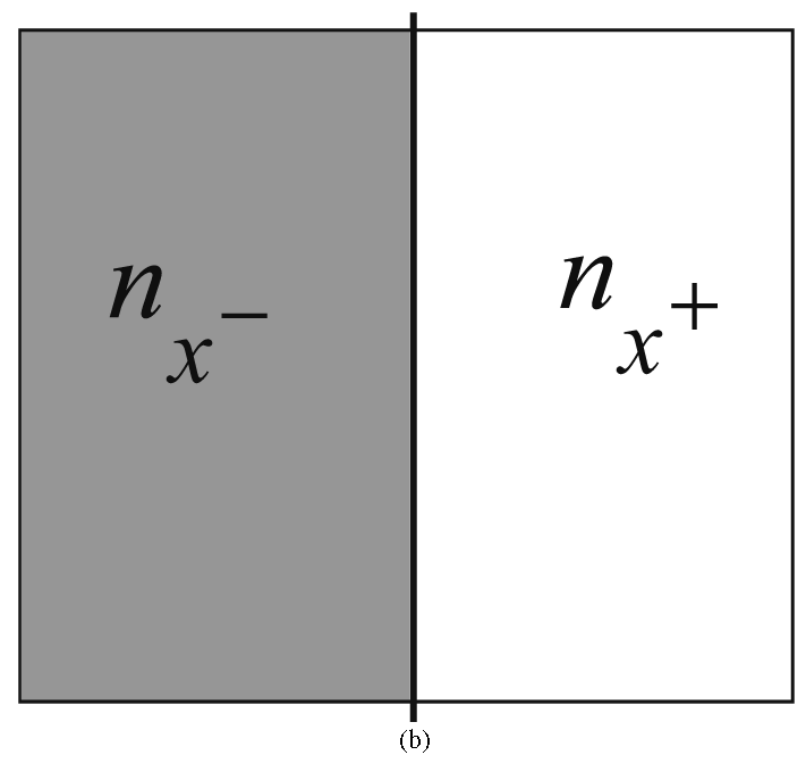

Fig. 1. Interfaces of dielectric materials. (a) Horizontal interface. (b) Vertical interface.

tively. Similarly, for a vertical interface as shown in Fig. 1(b), we have

$$
\left.n_{x+}^{2} \frac{\partial H_{y}}{\partial x}\right|_{x-}-\left.n_{x-}^{2} \frac{\partial H_{y}}{\partial x}\right|_{x+}=\left(n_{x+}^{2}-n_{x-}^{2}\right) \frac{\partial H_{x}}{\partial y}
$$

and

$$
\left.\frac{\partial H_{x}}{\partial x}\right|_{x+}=\left.\frac{\partial H_{x}}{\partial x}\right|_{x-}
$$

where $x+$ and $x-$ are referred to the locations at the infinitesimally right and left of the vertical interface, respectively. In summary, we construct the novel full-vectorial mode solver based

$$
\begin{aligned}
& \frac{\partial^{2} H_{x}}{\partial x^{2}}+\frac{\partial^{2} H_{x}}{\partial y^{2}}+k_{0}^{2}\left(n^{2}-n_{\text {eff }}^{2}\right) H_{x}-\frac{1}{n^{2}} \frac{\partial n^{2}}{\partial y} \frac{\partial H_{x}}{\partial y}+\frac{1}{n^{2}} \frac{\partial n^{2}}{\partial y} \frac{\partial H_{y}}{\partial x}=0 \\
& \frac{\partial^{2} H_{y}}{\partial x^{2}}+\frac{\partial^{2} H_{y}}{\partial y^{2}}+k_{0}^{2}\left(n^{2}-n_{\text {eff }}^{2}\right) H_{y}-\frac{1}{n^{2}} \frac{\partial n^{2}}{\partial x} \frac{\partial H_{y}}{\partial x}+\frac{1}{n^{2}} \frac{\partial n^{2}}{\partial x} \frac{\partial H_{x}}{\partial y}=0
\end{aligned}
$$


on the MPM from (4)-(9). The full-vectorial version of MPM derived above can deal with the more complicated devices considering polarization dependence and coupling and can certainly offer a more comprehensive modal analysis.

\section{NumericAl TECHNIQUES}

\section{A. MPM and Interfacial Conditions}

In this paper, the transverse magnetic field components $H_{x}$ and $H_{y}$ in subdomain $e$ are represented by a product of the cardinal basis functions [10] $\theta(x)$ of $N_{x}+1$ terms in the $x$ direction and $\psi(y)$ of $N_{y}+1$ terms in the $y$ direction, and the grid point values of $H_{x}$ and $H_{y}$ fields at $\left(N_{x}+1\right) \times\left(N_{y}+1\right)$ collocation points denoted as $H_{p, q}^{x, e}$ and $H_{p, q}^{y, e}$ as follows:

$$
H_{x}^{e}(x, y)=\sum_{p=0}^{N_{x}} \sum_{q=0}^{N_{y}} \theta_{p}^{e}(x) \psi_{q}^{e}(y) H_{p, q}^{x, e}
$$

and

$$
H_{y}^{e}(x, y)=\sum_{p=0}^{N_{x}} \sum_{q=0}^{N_{y}} \theta_{p}^{e}(x) \psi_{q}^{e}(y) H_{p, q}^{y, e}
$$

where $\theta_{j}^{e}\left(x_{i}\right)=\delta_{i j}$ and $\psi_{j}^{e}\left(y_{i}\right)=\delta_{i j}$, and $\delta_{i j}$ denotes the Kronecker delta. The computations are directly operated in physical space $H_{p, q}^{x, e}$ and $H_{p, q}^{y, e}$ to obtain the field distributions instead of in spectral space, which need additional computational efforts by the Fourier transformation. We substitute (10) and (11) into the differential (4) and (5) and demand that (4) and (5) are perfectly satisfied at these $\left(N_{x}-1\right) \times\left(N_{y}-1\right)$ collocation points [11]. The remainder two points in each direction not included for subdomain $e$ are replaced by satisfying (6) and (7), and (8) and (9), respectively, at the horizontal and vertical interfaces. The differential equations are thus converted to a matrix eigenvalue equation as follows:

$$
\left[\begin{array}{ll}
S & 0 \\
0 & S
\end{array}\right]\left[\begin{array}{c}
H_{x}^{e} \\
H_{y}^{e}
\end{array}\right]=\beta^{2}\left[\begin{array}{c}
H_{x}^{e} \\
H_{y}^{e}
\end{array}\right]
$$

where

$$
\begin{aligned}
S H_{r}^{e}= & \left.\sum_{i=1}^{N_{x}} \sum_{j=1}^{N_{y}}\left[\frac{\partial^{2} H_{r}^{e}}{\partial x^{2}}+\frac{\partial^{2} H_{r}^{e}}{\partial y^{2}}+k_{0}^{2} n^{2} H_{r}^{e}\right]\right|_{x=x_{i}, y=y_{j}} \\
= & \sum_{i=1}^{N_{x}-1} \sum_{j=1}^{N_{y}-1}\left[\sum _ { p = 0 } ^ { N _ { x } } \sum _ { q = 0 } ^ { N _ { y } } \left\{\theta_{p}^{(2)}(x) \psi_{q}(y)+\theta_{p}(x) \psi_{q}^{(2)}(y)\right.\right. \\
& \left.\left.+k_{0}^{2} n^{2}(x, y) \theta_{p}(x) \psi_{q}(y)\right\}\right]\left.\right|_{\substack{x=x_{i}, y=y_{j} \\
(13)}}
\end{aligned}
$$

and the continuity conditions from (6) to (9) at all interfacial points shared with adjacent subdomains are given in the form of a matrix

$$
[\mathbf{C}][\mathbf{H}]=\left[\begin{array}{ll}
\mathbf{C}_{11} & \mathbf{C}_{12} \\
\mathbf{C}_{21} & \mathbf{C}_{12} \\
\mathbf{C}_{31} & \mathbf{C}_{32} \\
\mathbf{C}_{41} & \mathbf{C}_{42}
\end{array}\right]\left[\begin{array}{l}
H_{x}^{e} \\
H_{y}^{e}
\end{array}\right]=\mathbf{0}
$$

where

$$
\begin{aligned}
& \mathbf{C}_{\mathbf{1 1}}=\left.\sum_{i=0}^{N x}\left(n_{y+}^{2} \sum_{p=0}^{N_{x}} \sum_{q=0}^{N_{y}} \theta_{p}(x) \psi_{q}^{(1)}(y)\right]\right|_{x=x_{i}, y=y_{-}} \\
& \left.\left.-n_{y-}^{2} \sum_{p=0}^{N_{x}} \sum_{q=0}^{N_{y}} \theta_{p}(x) \psi_{q}^{(1)}(y)\right]\left.\right|_{x=x_{i}, y=y_{+}}\right) \\
& \mathbf{C}_{\mathbf{1 2}}=-\left(n_{y+}^{2}-n_{y-}^{2}\right) \\
& \times\left.\sum_{j=0}^{N y}\left[\sum_{p=0}^{N_{x}} \sum_{q=0}^{N_{y}} \theta_{p}^{(1)}(x) \psi_{q}(y)\right]\right|_{x=x_{+}, y=y_{j}} \\
& \mathbf{C}_{21}=\mathbf{0} \text {, } \\
& \mathbf{C}_{\mathbf{2 2}}=\left.\sum_{i=0}^{N x}\left(\sum_{p=0}^{N_{x}} \sum_{q=0}^{N_{y}} \theta_{p}(x) \psi_{q}^{(1)}(y)\right]\right|_{x=x_{i}, y=y_{+}} \\
& \left.\left.-\sum_{p=0}^{N_{x}} \sum_{q=0}^{N_{y}} \theta_{p}(x) \psi_{q}^{(1)}(y)\right]\left.\right|_{x=x_{i}, y=y_{-}}\right) \\
& \mathbf{C}_{\mathbf{3 1}}=\left(n_{x+}^{2}-n_{x-}^{2}\right) \\
& \times\left.\sum_{i=0}^{N x}\left[\sum_{p=0}^{N_{x}} \sum_{q=0}^{N_{y}} \theta_{p}(x) \psi_{q}^{(1)}(y)\right]\right|_{x=x_{i}, y=y_{+}} \\
& \mathbf{C}_{\mathbf{3 2}}=\sum_{j=0}^{N y}\left(n_{x}^{2}-\left.\sum_{p=0}^{N_{x}} \sum_{q=0}^{N_{y}} \theta_{p}^{(1)}(x) \psi_{q}(y)\right|_{x=x_{+}, y=y_{j}}\right. \\
& \left.-n_{x}^{2}+\left.\sum_{p=0}^{N_{x}} \sum_{q=0}^{N_{y}} \theta_{p}^{(1)}(x) \psi_{q}(y)\right|_{x=x_{-}, y=y_{j}}\right) \\
& \mathbf{C}_{\mathbf{4 1}}=\sum_{j=0}^{N y}\left(\left.\sum_{p=0}^{N_{x}} \sum_{q=0}^{N_{y}} \theta_{p}^{(1)}(x) \psi_{q}(y)\right|_{x=x_{+}, y=y_{j}}\right. \\
& \left.-\left.\sum_{p=0}^{N_{x}} \sum_{q=0}^{N_{y}} \theta_{p}^{(1)}(x) \psi_{q}(y)\right|_{x=x_{-}, y=y_{j}}\right)
\end{aligned}
$$

In (13) and (14), $\theta_{p}^{(h)}(x)$ and $\psi_{q}^{(h)}(y)$ represent the $h$ th order derivatives of cardinal basis functions $\theta_{p}(x)$ and $\psi_{q}(y)$, respectively. By assembling all subdomains, the propagation constants and the corresponding unknown fields $H_{x}$ and $H_{y}$ can be obtained by a generalized eigenvalue solver.

\section{B. Determination of the Cardinal Basis Functions and Scaling Factor}

According to the feature of computational intervals, Chebyshev polynomials and the Fourier series are the two candidates for finite extension; the former are robust to nonperiodic functions, while the latter are suitable to periodic ones. For unbounded extension, the characteristic of electromagnetic fields decays exponentially as the independent variable tends to infinity; hence, HG or Laguerre-Gauss (LG) functions are the most favorable ones for the intervals $(-\infty, \infty)$ and $[0, \infty)$, respectively. Because of the product of a decaying exponent 


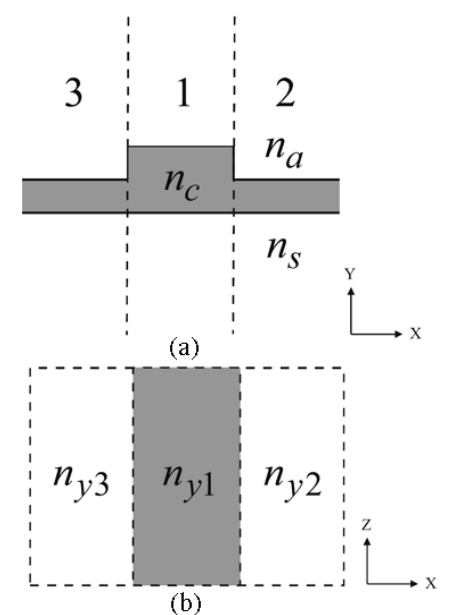

Fig. 2. (a) Cross section of a rib waveguide. (b) Equivalent slab waveguide to original rib waveguide after using EIM along the $y$ direction.

with Hermite or Laguerre polynomials, the electromagnetic fields are well coincided, and no real enclosed computational window or specific boundary condition is needed to assign. In this paper, Chebyshev polynomials are applied to the finite extension and LG functions are utilized in the semi-infinite one. When Chebyshev polynomials are used as basis functions, the explicit form of cardinal basis function $\theta_{p}(x)$ [10] can be expressed as

$$
\theta_{p}(x)=\frac{(-1)^{p+1}\left(1-x^{2}\right) T_{N}^{\prime}(x)}{c_{p} N^{2}\left(x-x_{p}\right)}, \quad x \neq x_{p}
$$

where $T_{N}(x)$ denotes the Chebyshev polynomial of order $N$, $c_{0}=c_{N}=2, c_{p}=1(1 \leq p \leq N-1)$, and the prime denotes the first derivative with respect to $x$. In regard to the collocation points, we choose Chebyshev Gauss-Lobatto points. As for the LG functions, cardinal basis function are given by

$$
\theta_{p}(\alpha x)=\frac{e^{-\alpha x / 2}}{e^{-\alpha x_{p} / 2}} \frac{\alpha x L_{N}(\alpha x)}{\left(\alpha x L_{N}\right)^{\prime}\left(\alpha x_{p}\right)\left(\alpha x-\alpha x_{p}\right)}, \quad x \neq x_{p}
$$

where $L_{N}(\alpha x)$ denotes Laguerre polynomials of order $N$. In (16), $\alpha$ is a so-called scaling factor to be determined, and it may influence the accuracy for a given terms of basis functions.

For a given $N, \alpha$ is usually picked up through a few trial and errors with additional expense of a considerable amount of computational effort [7]. In the present method, we combine two techniques, one from Tang [14] and the other, effective index method (EIM), from [13] to provide a reliable and fast method to determine $\alpha$. The detailed derivations have been discussed in our previous paper [12]. For a given number of $N$ basis functions, the optimum $\alpha$ can be obtained through the following relationship:

$$
\alpha=\max _{0 \leq j \leq N} \frac{\left\{x_{j}\right\}}{M}
$$

where $M$ indicates the finite support and $x_{j}$ is the collocation point at position $j$. To determine $M$, the effective index $n_{\text {eff }}$ and the decay rate of the highest order guided mode has to be obtained in advance, then $M$ can be estimated subsequently. We choose EIM to compute $n_{\text {eff }}$ because of its simplicity and fast

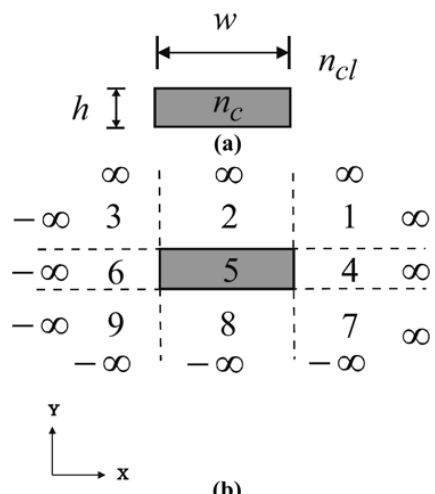

(b)

Fig. 3. (a) Cross section of rectangular channel waveguide with index of core $n_{c}$ surrounded by the cladding with index of $n_{c l}$. (b) The division of computational domain of the rectangular channel waveguide.

obtainment in spite of the method is not accurate enough. As illustrated in Fig. 2(a), the vertical dotted lines split the rib waveguide into three subregions. Using the dispersion relation of the three-layer waveguide in the $y$ direction, one determines the effective indexes of subregions 1,2 , and 3 as $n_{y 1}, n_{y 2}$, and $n_{y 3}$ in Fig. 2(b), respectively. If the cutoff condition is achieved in subregion 2 or 3 , then $n_{y 2}$ and $n_{y 3}$ are assigned by $n_{s}$. The dispersion relation is utilized again in the $x$ direction to obtain the equivalent effective index $n_{\text {eq }}$ to the original waveguide structure. The decay rates along the $y$ direction extending to the substrate side for the three subregions are given by

$$
\gamma_{y s i}=k_{0} \sqrt{n_{y i}^{2}-n_{s}^{2}}, \quad i=1,2,3
$$

and those extending to the air side are

$$
\gamma_{y a i}=k_{0} \sqrt{n_{y i}^{2}-n_{a}^{2}}, \quad i=1,2,3
$$

where $n_{s}$ and $n_{a}$ denote the refractive indexes of substrate and air, respectively. Similarly, the decay rates along the $x$ direction are

$$
\gamma_{x j}=k_{0} \sqrt{n_{\text {eq }}^{2}-n_{y j}^{2}}, \quad j=2 \text { or } 3
$$

where $n_{\text {eq }}$ is the effective index of the original waveguide. Accordingly, we can estimate the finite support along the $y$ direction according to [15] by

$$
\begin{aligned}
\frac{H\left(y_{t}+M_{y s i}\right)}{H\left(y_{t}\right)} & =\exp \left[-\int_{y_{t}}^{y_{t}+M_{y s i}} \gamma_{y s i} d y\right]=\kappa \\
i & =1,2,3
\end{aligned}
$$

and

$$
\begin{aligned}
\frac{H\left(y_{t}+M_{y a i}\right)}{H\left(y_{t}\right)} & =\exp \left[-\int_{y_{t}}^{y_{t}+M_{y a i}} \gamma_{y a i} d y\right]=\kappa \\
i & =1,2,3
\end{aligned}
$$

and that along the $x$ direction $M_{x j}$ by

$$
\begin{aligned}
\frac{H\left(x_{t}+M_{x j}\right)}{H\left(x_{t}\right)} & =\exp \left[-\int_{x_{t}}^{x_{t}+M_{x j}} \gamma_{x j} d x\right]=\kappa \\
j & =2 \text { or } 3
\end{aligned}
$$

where $x_{t}$ and $y_{t}$ are the turning points in the $x$ and $y$ directions, respectively. As shown in (21)-(23), the parameter $\kappa$ denotes the 


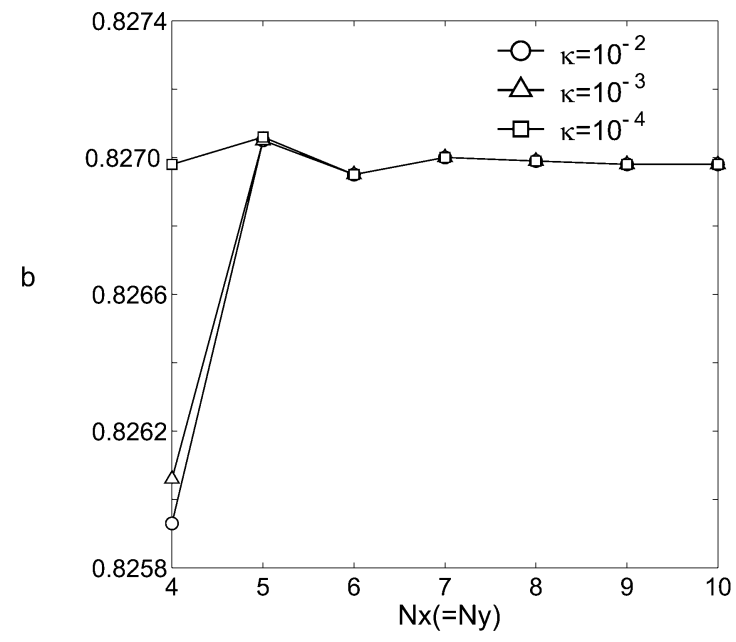

(a)

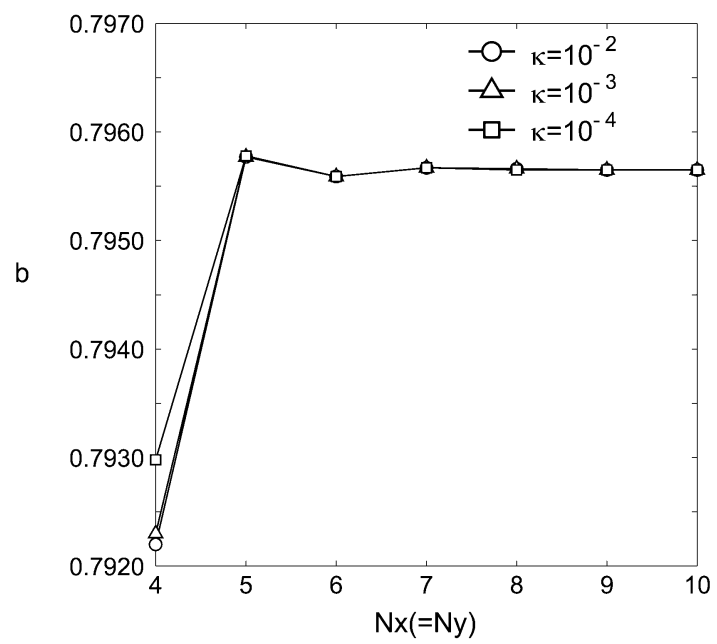

(b)

Fig. 4. Convergence of solutions for the (a) $H_{11}^{y}$ and (b) $H_{11}^{x}$ modes in the rectangular channel waveguide for different $\kappa$.

ratio of the field strength at the position of turning points plus $M$ relative to that at turning points. For simplicity, we let $\kappa$ be equal in both the $x$ and $y$ directions. All of the numerical examples computed in Section IV are chosen as $\kappa=10^{-2}$. However, $\kappa$ is insensitive even though $\kappa=10^{-4}$ is used.

\section{NUMERICAL RESULTS}

To validate the performances of the present method, the dispersion relations of rectangular channel and semiconductor rib waveguides of practical interest are analyzed. We also discuss the effect for setting different $\kappa$ in (21)-(23). In addition, the computational time is also provided for the rib waveguide. Here, the corners yield no singularity due to the magnetic field formulations used. However, the singular effect is exhibited apparently at a dielectric corner while the wave equations are formulated by the electric fields. Because the dielectric will affect the static electric field but does not affect the static magnetic field, unless the dielectric is replaced by a magnetizable medium, and the electric field at the dielectric corner within an extremely small region can be considered as a static electric field, the electric field can therefore exhibit a singular behavior [16]. As a result, the singularity has to be handled properly (i.e., the electric field

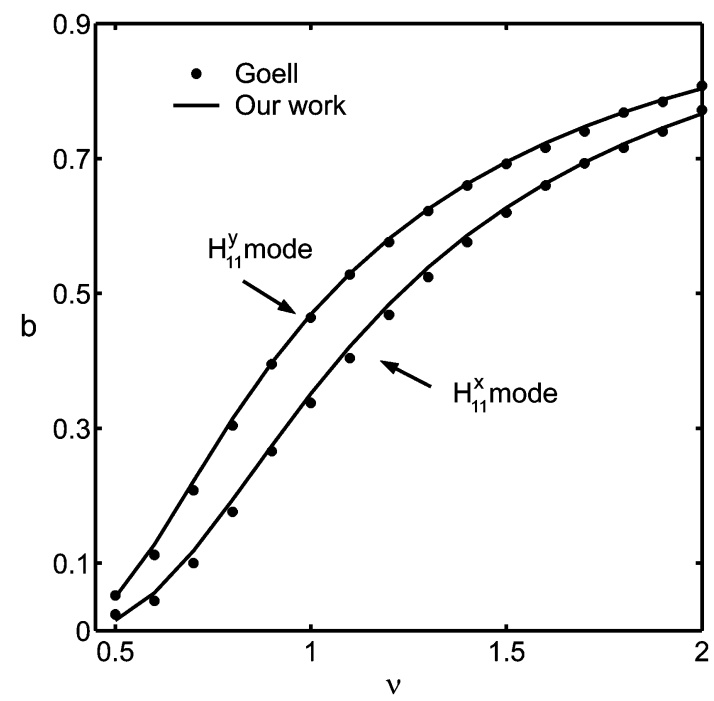

Fig. 5. Normalized propagation constant $b$ of the rectangular channel waveguide for lowest order $H^{y}\left(H_{11}^{y}\right)$ and $H^{x}\left(H_{11}^{x}\right)$ modes versus the normalized frequency $\nu$ obtained by our work (solid lines) and that calculated by Goell's method (filled circles) [19].

at corners can be approximated as the gradient of a scalar potential function [17], [18]), otherwise the results calculated by numerical methods will show poor convergence.

\section{A. Rectangular Channel Waveguide}

Channel waveguides are a class of comprehensive photonic devices in optical communications. An exact analytical solution cannot be obtained due to the presence of corners. Many numerical schemes have studied the problem in past few decades. Among these calculations, the results estimated by Goell [19] are so far regarded as a benchmark results for comparing the accuracy. As a result, we take an example, which has been computed by Goell to test the accuracy of our method. Considering a waveguide structure with the cross section and the domain decomposition (DD) division is depicted, respectively, in Fig. 3(a) and (b). The width and height of the core region with index of $n_{c}=1.5$ surrounded by cladding with index of $n_{c l}=1.0$ are $w=3 \mu \mathrm{m}$ and $h=w / 2$, respectively. In Fig. 3(b), the core region is indicated as subdomain 5 , which is consisted of finite extension in both the $x$ and $y$ directions; the remaining subdomains possess at least one semi-infinite extension. For instance, subdomains 4 and 6 have semi-infinite extension in the $x$ direction and finite in the $y$ direction. The subdomains $1,3,7$, and 9 have semi-infinite extension in both transverse directions. According to our consideration [12], the finite extension and the semi-infinite one are, respectively, expanded by Chebyshev polynomials and LG functions with the a priori determined scaling factor. After substituting suitable functions to the corresponding subdomains based on (4) and (5), the interfacial conditions (6)-(9) are applied to incorporate subdomains.

The convergent behaviors of the normalized propagation constant $b=\left(n_{\text {eff }}^{2}-n_{c l}^{2}\right) /\left(n_{c}^{2}-n_{c l}^{2}\right)$ varying with the number of the basis function $N_{x}\left(=N_{y}\right)$ in each subdomain under different chosen $\kappa$ using the present method for the lowest order $H^{y}$ and $H^{x}$ modes operated in wavelength $\lambda=1.55 \mu \mathrm{m}$ are shown in Fig. 4(a) and (b), respectively. For the relationship between the number of the basis function and the mesh grid in this example, it can be expressed by the form of $\left(N_{x} \times 3-2\right) \times\left(N_{y} \times 3-2\right)$. To 


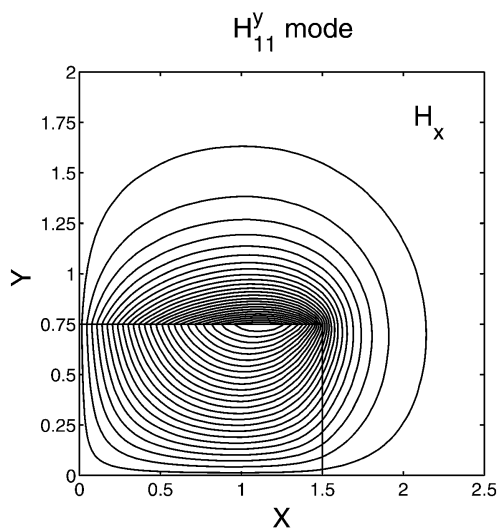

(a)

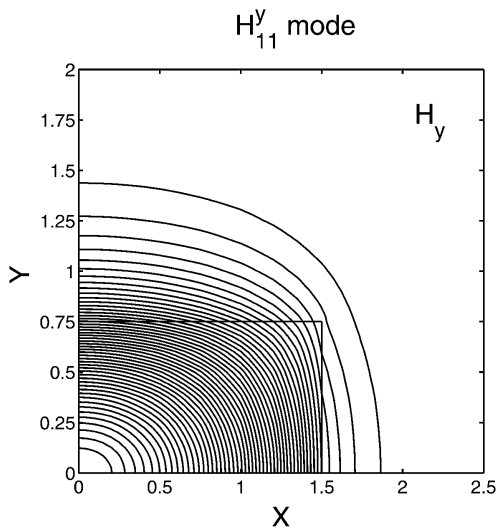

(b)

Fig. 6. Contours of magnetic field profiles for the $H_{11}^{y}$ mode of the rectangular channel waveguide. (a) $x$ component (minor field) $H_{x}$. (b) $y$ component (major field) $H_{y}$.

beyond eight terms of basis function (the mesh grid is $22 \times 22$ ), the calculated values of $H_{11}^{y}$ and $H_{11}^{x}$ modes converge, respectively, close to the values 0.8270 and 0.7956 , and the difference for further denser mesh is to be smaller than the order of $10^{-4}$ as shown. The finite supports $M\left(\kappa=10^{-2}\right)$ in the $\pm y$ directions are $\pm 1.11 \mu \mathrm{m}$ and are $\pm 1.43 \mu \mathrm{m}$ in the $\pm x$ directions. The scaling factors are $\alpha_{x}=16.97$ in the $x$ direction and $\alpha_{y}=17.46$ in the $y$ direction under $N_{x}=N_{y}=8$ used, accordingly. We can see that $\kappa$ in Fig. 4(a) and (b) illustrates slight influence to $b$. The dispersion relations of the modes $H_{11}^{y}$ and $H_{11}^{x}$ in terms of $b$ and the normalized frequency $\nu=k_{0} h \sqrt{n_{c}^{2}-n_{c l}^{2}} / \pi$ obtained by both Goell [19] and our work are shown in Fig. 5. In each subdomain, eight terms of basis functions are used in both the $x$ and $y$ directions, i.e., $N_{i x}=N_{i y}=8, i=1-9$. Fig. 5 shows that our results are in excellent agreement with Goell's solutions. The calculated contours operated in wavelength $\lambda=1.55 \mu \mathrm{m}$ (i.e., $v=2.164$ ) of the magnetic field components $H_{x}$ and $H_{y}$ for the lowest order mode $H_{11}^{y}$ are shown in Fig. 6(a) and (b). As to the mode $H_{11}^{x}$, Fig. 7(a) and (b) illustrate $H_{x}$ and $H_{y}$ components, respectively. Because of the symmetric structure of the waveguide, only one-quarter of the field profiles are displayed. In addition, the discontinuous features of the derivatives of magnetic field components $H^{x}$ and $H^{y}$ at horizontal and vertical interfaces, respectively, are also clearly exhibited in Figs. 6 and 7.

Additionally, to demonstrate the reliable of the present method applying to waveguides while the high-order modes

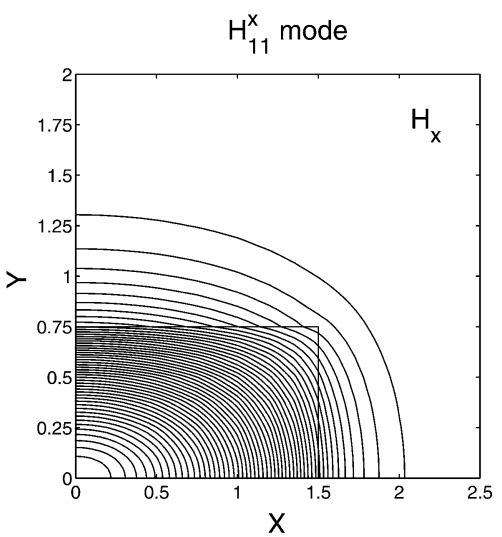

(a)

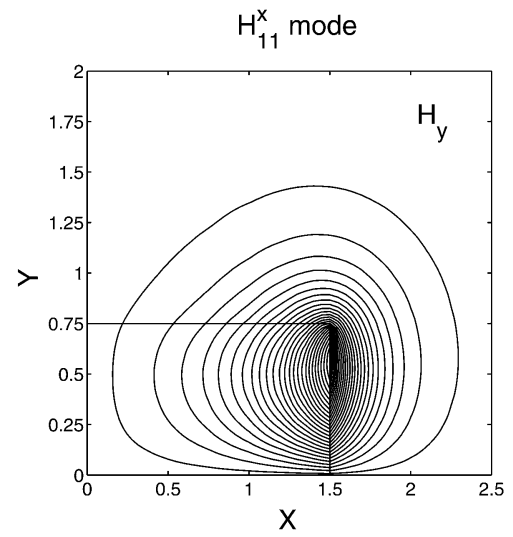

(b)

Fig. 7. Contours of magnetic field profiles for the $H_{11}^{x}$ mode of the rectangular channel waveguide. (a) $x$ component (major field) $H_{x}$. (b) $y$ component (minor field) $H_{y}$.

TABLE I

Convergence of the Normalized Propagation Constant Versus the Basis FunCtions $N$ USED IN EACH SUbDOMAIN FOR THE FIRST FOUR MOdES OF THE RECTANGULAR CHANNEL WAVEGUIDE WITH $\kappa=10^{-2}$

\begin{tabular}{c|c|c|c|c|c}
\hline Mode & $\mathrm{N}=6$ & $\mathrm{~N}=8$ & $\mathrm{~N}=10$ & $\mathrm{~N}=12$ & Goell [19] \\
\hline \hline $\mathrm{H}_{11}^{\mathrm{y}}$ & 0.5842 & 0.5812 & 0.5811 & 0.5810 & 0.580 \\
$\mathrm{H}_{11}^{\mathrm{x}}$ & 0.4859 & 0.4838 & 0.4838 & 0.4837 & 0.472 \\
$\mathrm{H}_{21}^{\mathrm{y}}$ & 0.2296 & 0.2290 & 0.2285 & 0.2283 & 0.220 \\
$\mathrm{H}_{21}^{\mathrm{x}}$ & 0.2079 & 0.2054 & 0.2048 & 0.2045 & 0.202 \\
\hline
\end{tabular}

TABLE II

SAME as TABle I, Except That the Results ARE CALCULATED AT $\kappa=10^{-3}$

\begin{tabular}{c|c|c|c|c|c}
\hline Mode & $\mathrm{N}=6$ & $\mathrm{~N}=8$ & $\mathrm{~N}=10$ & $\mathrm{~N}=12$ & Goell [19] \\
\hline $\mathrm{H}_{11}^{\mathrm{y}}$ & 0.5929 & 0.5814 & 0.5811 & 0.5810 & 0.580 \\
$\mathrm{H}_{11}^{\mathrm{x}}$ & 0.4936 & 0.4840 & 0.4837 & 0.4837 & 0.472 \\
$\mathrm{H}_{21}^{\mathrm{y}}$ & 0.2357 & 0.2287 & 0.2283 & 0.2281 & 0.220 \\
$\mathrm{H}_{21}^{\mathrm{x}}$ & 0.2137 & 0.2058 & 0.2049 & 0.2046 & 0.202 \\
\hline
\end{tabular}

exist, the normalized frequency $\nu=1.2$ is considered. In this example, the structure is able to support four modes in order, $H_{11}^{y}, H_{11}^{x}, H_{21}^{y}$, and $H_{21}^{x}$. The convergence of the normalized propagation constants as a function of the terms of basis functions $N\left(=N_{x}=N_{y}\right)$ calculated by our method and the data obtained by [19] are shown in Tables I-III under $\kappa=10^{-2}$, $\kappa=10^{-3}$, and $\kappa=10^{-4}$, respectively. The finite supports $M$ for the three different $\kappa$ are about 14,20 , and $27 \mu \mathrm{m}$, 
TABLE III

SAME AS TABle I, EXCEPT THAT THE RESUltS ARE CALCULATED AT $\kappa=10^{-4}$

\begin{tabular}{c|c|c|c|c|c}
\hline Mode & $\mathrm{N}=6$ & $\mathrm{~N}=8$ & $\mathrm{~N}=10$ & $\mathrm{~N}=12$ & Goell [19] \\
\hline $\mathrm{H}_{11}^{\mathrm{y}}$ & 0.6121 & 0.5831 & 0.5811 & 0.5810 & 0.580 \\
$\mathrm{H}_{11}^{\mathrm{x}}$ & 0.5099 & 0.4851 & 0.4837 & 0.4837 & 0.472 \\
$\mathrm{H}_{21}^{\mathrm{y}}$ & 0.2499 & 0.2295 & 0.2281 & 0.2279 & 0.220 \\
$\mathrm{H}_{21}^{\mathrm{x}}$ & 0.2287 & 0.2069 & 0.2050 & 0.2046 & 0.202 \\
\hline
\end{tabular}

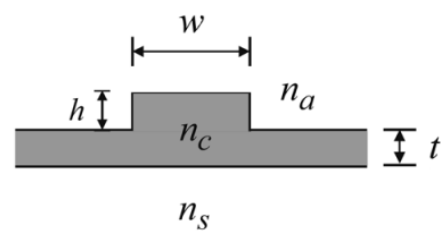

(a)

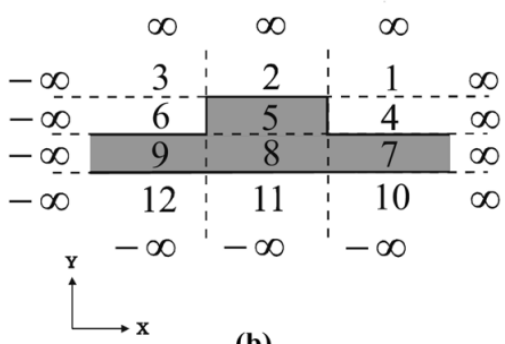

(b)

Fig. 8. (a) Cross section of a rib waveguide with refractive indexes of core $n_{c}$, substrate $n_{s}$, and air $n_{a}$. (b) Division of computational domain of the rib waveguide.

respectively. It can be observed that the convergences while considering different orders of $\kappa$ seem to be insensitive for the first four modes as shown in Tables I-III. Hence, for the waveguides supporting high-order modes, the present full-vectorial mode solver can still keep efficient and accurate advantages. In our derivation, the spreading $M$ of field is determined by the chosen $\kappa$. According to our numerical experimental data and physical meaning, the choice of $10^{-2}<\kappa<10^{-4}$ is suitable and reasonable. In the present scheme for determining $\kappa$, the smaller value of $\kappa$ means that the farther spreading of the optical field is considered for computing, and thus more collocation points (i.e., the number of basis functions) are required. In general, for the strongly guiding waveguide, the larger value of $\kappa=\left(10^{-2}\right)$ can be chosen for saving the computational effort. As for the weakly guiding one, we use the larger $\kappa=10^{-4}$ for keeping accuracy.

\section{B. Semiconductor Rib Waveguide}

The cross section and the division of a typical rib waveguide, which has been analyzed by several researchers [20], [21], are sketched in Fig. 8(a) and (b), respectively. The relevant parameters are set to $w=3 \mu \mathrm{m}, h+t=1 \mu \mathrm{m}, n_{c}=3.44, n_{s}=3.4$, $n_{a}=1.0$, at wavelength $\lambda=1.15 \mu \mathrm{m}$. The slab width $t$ varies from 0.1 to $0.9 \mu \mathrm{m}$. The finite supports $M$ for different $t$ are estimated by EIM. For instance, the finite support in the $y$ direction for subdomain $2, M_{y 2}$ (under $\kappa=10^{-2}$ ) is $0.26 \mu \mathrm{m}$ for $H_{11}^{y}$ and $H_{11}^{x}$ modes (we set $M_{y 2}=M_{y 1}=M_{y 3}$ ), and $M_{y 11}$ is 2.47 and $2.60 \mu \mathrm{m}$ for $H_{11}^{y}$ and $H_{11}^{x}$ modes (likewise, $M_{y 11}=M_{y 10}=$ $M_{y 12}$ ), respectively, when $t=0.5 \mu \mathrm{m}$. In the $x$ direction, $M_{x 1}$ is $2.70 \mu \mathrm{m}$ for $H_{11}^{y}$ and $2.87 \mu \mathrm{m}$ for $H_{11}^{x}$ modes. Moreover, the relationships $M_{x 1}=M_{x 4}=M_{x 7}=M_{x 10}$ and $M_{x 3}=$

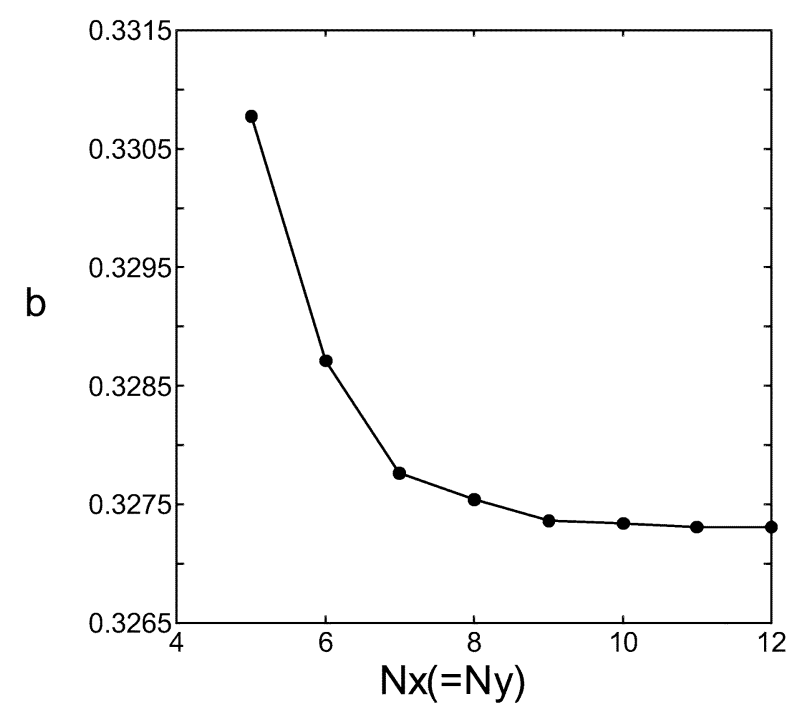

(a)

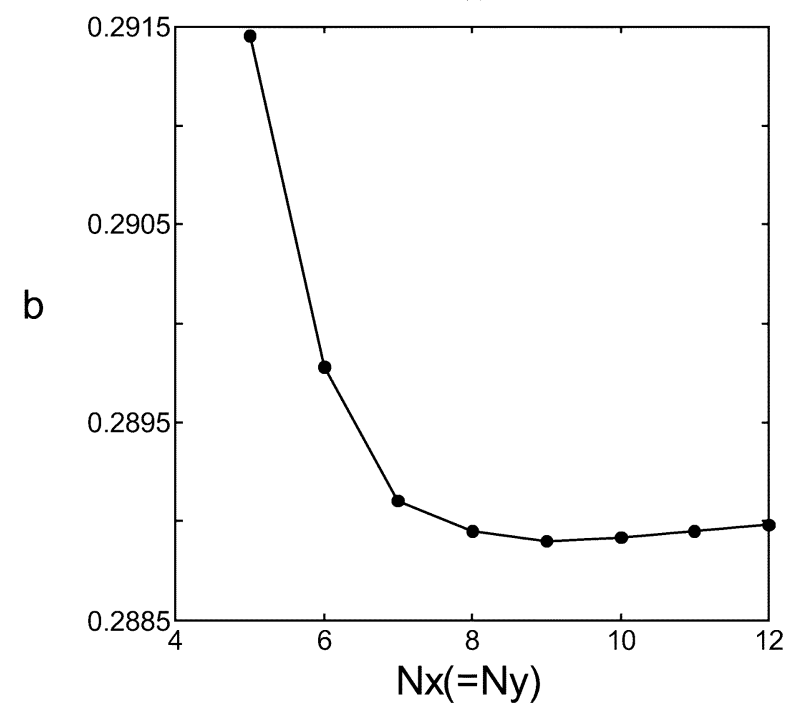

(b)

Fig. 9. Convergence of solutions of the (a) $H_{11}^{y}$ and (b) $H_{11}^{x}$ modes for the rib waveguide.

TABLE IV

NORMALIZED PRopagation CONSTANT OF $H_{11}^{y}$ MODE OF THE RiB WAVEGUIDE FOR DifFERENT SLAB WidTh $t$ COMPUTED BY VARIOUS Methods. MPM IS THE PRESENT METHOD USING TEN TERMS OF Basis FunCtIONS IN EACH SUBdomain

\begin{tabular}{c|c|c|c|c}
\hline$t(\mu \mathrm{m})$ & MMM [20] & FEM [20] & FD-BPM [21] & MPM \\
\hline \hline 0.1 & 0.3019 & 0.3019 & 0.3039 & 0.3020 \\
0.3 & 0.3111 & 0.3110 & 0.3144 & 0.3113 \\
0.5 & 0.3270 & 0.3270 & 0.3303 & 0.3273 \\
0.7 & 0.3512 & 0.3512 & 0.3533 & 0.3515 \\
0.9 & $*$ & $*$ & 0.3879 & 0.3884 \\
\hline
\end{tabular}

$M_{x 6}=M_{x 9}=M_{x 12}$ are constrained in our computations. The normalized propagation constant $b=\left(n_{\text {eff }}^{2}-n_{s}^{2}\right) /\left(n_{c}^{2}-n_{s}^{2}\right)$ varied with the number of the basis function $N_{x}\left(=N_{y}\right)$ for the $H_{11}^{y}$ and $H_{11}^{x}$ modes are shown in Fig. 9(a) and (b). The mesh grid in this example is expressed by the form of $\left(N_{x} \times 4-3\right) \times$ $\left(N_{y} \times 3-2\right)$. We observe that the difference of $b$ for further increasing the number of basis functions are to be smaller than the order of $10^{-4}$ and the convergent results of $H_{11}^{y}$ and $H_{11}^{x}$ modes are, respectively, 0.3273 and 0.2890 using ten basis functions 
TABLE $\mathrm{V}$

SAme as Table V, EXCEPT That the Results ARE OBTAINED FOR $H_{11}^{x}$ MODE

\begin{tabular}{c|c|c|c|c}
\hline$t(\mu \mathrm{m})$ & MMM [20] & FEM [20] & FD-BPM [21] & MPM \\
\hline \hline 0.1 & 0.2675 & 0.2675 & 0.2690 & 0.2674 \\
0.3 & 0.2751 & 0.2751 & 0.2776 & 0.2751 \\
0.5 & 0.2890 & 0.2890 & 0.2915 & 0.2890 \\
0.7 & 0.3107 & 0.3106 & 0.3127 & 0.3107 \\
0.9 & $*$ & $*$ & 0.3451 & 0.3455 \\
\hline
\end{tabular}

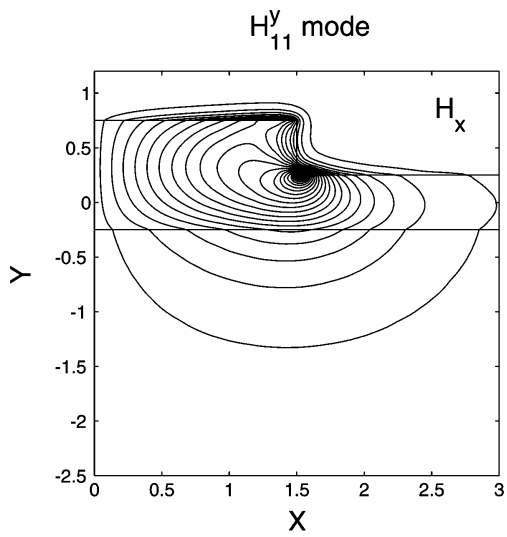

(a)

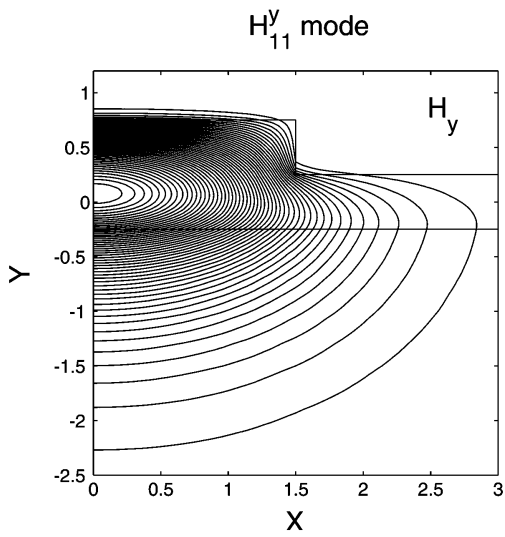

(b)

Fig. 10. Contours of magnetic field profiles for the $H_{11}^{y}$ mode of the rib waveguide. (a) $x$ component (minor field) $H_{x}$. (b) $y$ component (major field) $H_{y}$.

(the mesh grid is $37 \times 28$ ). The scaling factors are $\alpha_{y 2}=$ 101.44, $\alpha_{y 11}=10.68$, and $\alpha_{x 1}=9.77$ for $H_{11}^{y}$ mode and are $\alpha_{y 2}=101.44, \alpha_{y 11}=10.14$, and $\alpha_{x 1}=9.19$ for $H_{11}^{x}$ mode.

Tables IV and V present, respectively, the normalized propagation constants of $H_{11}^{y}$ and $H_{11}^{x}$ modes obtained by our method using ten basis functions in each subdomain together with that provided by other authors: the modal matching method (MMM) with 100-400 TE/TM mode pairs [20], the FE method employing about 2500 triangles of high-order $T_{15}$ edge elements [20], and the FD beam propagation method (FD-BPM) [21] for distinct $t$. Our results are in good agreement with the values obtained by MMM and FEM, especially; those of the $H_{11}^{x}$ mode are in virtually complete agreement. In addition, for the case of $t=0.5 \mu \mathrm{m}$, Fig. 10(a) and (b) show the contour plots of $H_{x}$ and $H_{y}$, respectively, for $H_{11}^{y}$ modes. Half of the profiles

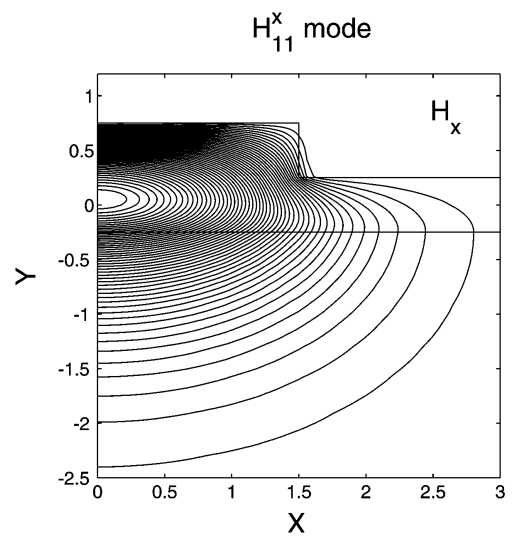

(a)

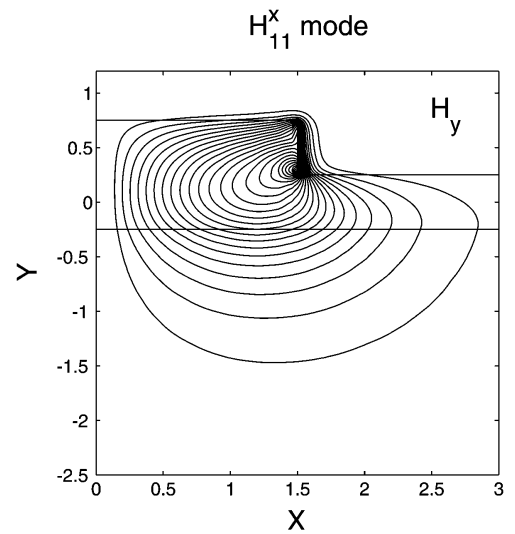

(b)

Fig. 11. Contours of magnetic field profiles for the $H_{11}^{x}$ mode of the rib waveguide. (a) $x$ component (major field) $H_{x}$. (b) $y$ component (minor field) $H_{y}$.

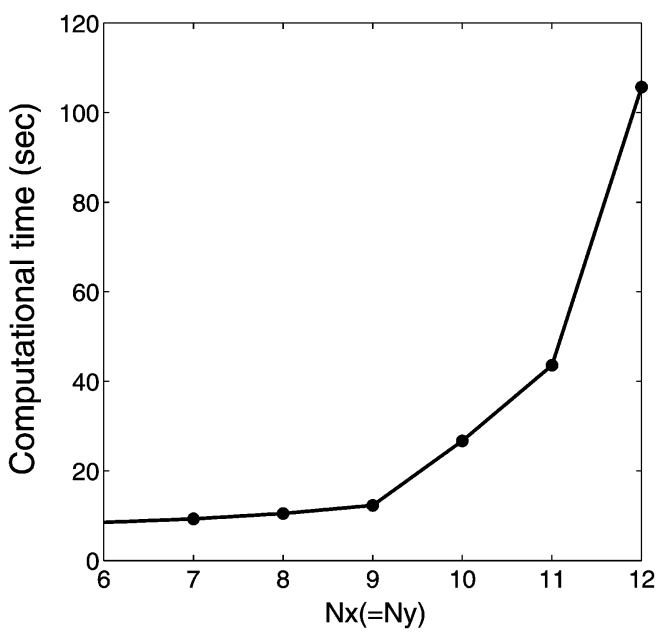

Fig. 12. Computational time as a function of the number of basis functions $N_{x}\left(=N_{y}\right)$ used in rib waveguide.

are displayed because of the symmetric structure with respect to $x=0$. Similarly, the contour plots of $H_{x}$ and $H_{y}$ for $H_{11}^{x}$ modes are shown in Fig. 11(a) and (b), respectively. Fig. 12 provides the computational time on a Pentium IV PC with a CPU clock rate of $2.8 \mathrm{GHz}$ in a double precision required by the MPM. It can be seen from the computational effort that the present method can be effortlessly implemented on a simple PC. 


\section{CONCLUSION}

A highly efficient and accurate full-vectorial mode solver in terms of TM fields for the analysis of dielectric optical waveguides with stepped index distribution has been presented. The present method is based on the pseudospectral method with DD. Merging the two techniques, namely, the DD and optimum scaling factor in LG functions, into the conventional pseudospectral method yields the present solution method accurate and efficient. Specially, the computational window is not explicitly assigned but implicitly involved in scaling factor. The performances have been demonstrated by solving numerical examples including rectangular channel and semiconductor rib wavegudes. The calculated results coincided well with Goell's solutions. In particular, the solutions of rib waveguides are compared with other available results and are shown in good agreement with mode matching and FE methods. The present method may be applied to more complicated photonic devices such as a directional coupler, modulator, filter and even dense wavelength division multiplexing. Although the formulations are at present based on the waveguides with material boundaries perpendicular to the $x$ or $y$ axis of rectangular coordinates, the general curvilinear representation will be derived in our future work to analyze more general devices of great interest.

\section{REFERENCES}

[1] K. S. Chiang, "Review of numerical and approximate methods for the modal analysis of general optical dielectric waveguides," Opt. Quantum Electron., vol. 26, pp. 113-134, Mar. 1994.

[2] M. S. Stern, "Semivectorial polarized $H$ field solutions for dielectric waveguides with arbitrary index profiles," IEE Proc. J., vol. 135, pp. 333-338, Oct. 1988.

[3] P. Lusse, P. Stuwe, J. Schule, and H. G. Unger, "Analysis of vectorial mode fields in optical waveguides by a new finite difference method," $J$. Lightw. Technol., vol. 12, pp. 487-493, Mar. 1994.

[4] B. M. A. Rahman and J. B. Davies, "Finite-element analysis of optical and microwave waveguide problem," IEEE Trans. Microw. Theory Tech., vol. MTT-32, no. 1, pp. 20-28, Jan. 1984.

[5] M. Koshiba, S. Maruyama, and K. Hirayama, "A vector finite element method with the high-order mixed-interpolation-type triangular elements for optical waveguiding problem," J. Lightw. Technol., vol. 12, no. 3, pp. 495-502, Mar. 1994.

[6] A. Sharma and S. Banerjee, "Method for propagation of total fields or beam through optical waveguides," Opt. Lett., vol. 14, pp. 94-96, Jan. 1989.

[7] S. Banerjee and A. Sharma, "Propagation characteristics of optical waveguiding structure by direct solution of the Helmholtz equation for total fields," J.Opt. Soc. Amer. A, vol. 6, pp. 1884-1894, Dec. 1989.

[8] A. Weisshaar, J. Li, R. L. Gallawa, and I. C. Goyal, "Vector and quasivector solutions for optical waveguide modes using efficient Galerkin's method with Hermite-Gauss basis functions," J. Lightw. Technol., vol. 13, no. 8, pp. 1795-1800, Aug. 1995.

[9] C. H. Henry and B. H. Verbeek, "Solution of the scalar wave equation for arbitrarily shaped dielectric waveguides by two-dimensional Fourier analysis," J. Lightw. Technol., vol. 7, pp. 308-313, Feb. 1989.

[10] J. P. Boyd, Lecture Notes in Engineering, Chebyshev and Fourier Spectral Methods, 2nd ed. Heidelberg, Germany: Springer-Verlag, 2000.

[11] C. Canuto, M. Y. Hussaini, A. Quarteroni, and T. A. Zang, Spectral Methods in Fluid Dynamics, ser. Springer Series in Computational Physics. Berlin, Germany: Springer-Verlag, 1988.

[12] C. C. Huang, C. C. Huang, and J. Y. Yang, "An efficient method for computing optical waveguides with discontinuous refractive index profiles using spectral collocation method with domain decomposition," $J$. Lightw. Technol., vol. 21, no. 10, pp. 2284-2296, Oct. 2003.
[13] T. Tamir, Guides-Wave Optoelectronics. Berlin, Germany: SpringerVerlag, 1988

[14] T. Tang, "The hermite spectral method for Gauss-type functions," SIAM J. Sci. Comput., vol. 14, pp. 594-605, May 1993.

[15] N. Ramanujam, L. Li, J. J. Burke, and M. A. Gribbons, "Determination of the truncation order and numerical window for modeling general dielectric waveguides by the Fourier method," J. Lightw. Technol., vol. 14, pp. 500-508, Mar. 1996.

[16] R. E. Collin, Field Theory of Guided Waves, 2nd ed, ser. IEEE/OUP Series on Electromagnetic Wave Theory. Oxford, U.K.: Oxford Univ. Press, 1990

[17] A. S. Sudbo, "Why are accurate computations of mode fields in rectangular dielectric waveguides difficult?," J. Lightw. Technol., vol. 16, no. 4, pp. 929-936, May 1992.

[18] W. W. Lui, C. L. Xu, W. P. Huang, and K. Yokoyama, "Full-vectorial mode analysis with considerations of field singularities at corners of optical waveguides," J. Lightw. Technol., vol. 17, no. 8, pp. 1509-1513, Aug. 1999

[19] J. E. Goell, "A circular harmonic computer analysis of rectangular waveguides," Bell. Syst. Tech. J., vol. 48, pp. 2133-2160, Sep. 1969.

[20] S. Selleri and J. Petráček, "Modal analysis of rib waveguide through finite element and mode matching methods," Opt. Quantum Electron. vol. 33, pp. 373-386, Apr./May 2001.

[21] W. P. Huang and C. L. Xu, "Simulation of three-dimensional optical waveguides by a full-vector beam propagation method," IEEE $J$. Quantum Electron., vol. 29, no. 10, pp. 2639-2649, Oct. 1993.

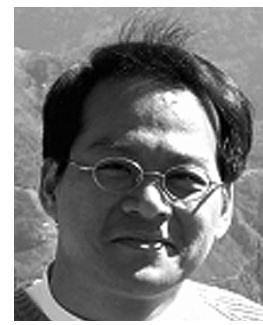

Chia-Chien Huang was born in I-Lan, Taiwan, R.O.C., on August, 21, 1972. He received the B.S. degree from the Department of Chemical Engineering, National Tsing-Hua University, Hsin-chu, Taiwan, R.O.C., in 1994 and the M.S. and Ph.D. degree from the Institute of Applied Mechanics, National Taiwan University, Taipei, Taiwan, R.O.C., in 1998 and 2003, respectively.

In August 2003, he joined the faculty of the Department of Information Management, Ling Tung College, Taichung, Taiwan, R.O.C., where he is currently an Assistant Professor. His current research interests are numerical methods for optical waveguide and photonic integrated circuits.

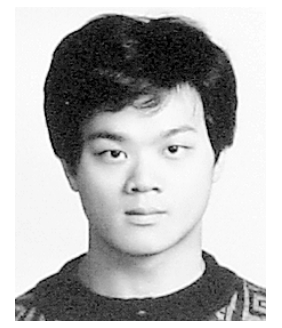

Chia-Chih Huang was born in I-Lan, Taiwan, R.O.C., on July 2, 1971. He received the B.S. degree in electrical engineering from National Taiwan University, Taipei, Taiwan, R.O.C., in 1993, the M.S. degree in electrical engineering from National Tsing-Hua University, Hsin-chu, Taiwan, R.O.C., in 1995, and the Ph.D. degree from the Graduate Institute of Electro-Optical Engineering, National Taiwan University, in 2003.

Since August 2003, he has been with the Department of Electronic Engineering, Tung Nan Institute of Technology, Taipei, where he is an Assistant Professor. His current research interests include modeling and design of passive integrated optical devices.

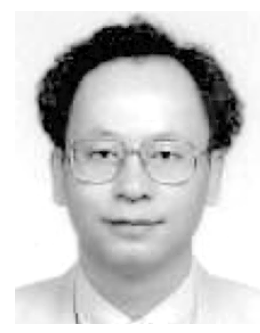

Jaw-Yen Yang received the Ph.D. degree from Stanford University, Stanford, CA, in 1983.

He was a National Research Council Associate at the NASA Ames Research Center. He is currently a professor in the Institute of Applied Mechanics at the National Taiwan University, Taiwan. His research focuses on computational modeling of electromagnetic wave interactions with complex structures, plasma stability, quantum hydrodynamics, and aerodynamics. 